\title{
Cisticercose suína, teníase e neurocisticercose humana no município de Barbalha, Ceará
}

\author{
[Porcine cysticercosis, taeniasis and human neurocysticercosis in municipal district of Barbalha, Ceará] \\ M.C. Silva ${ }^{1}$, A.A. Cortez ${ }^{1}$, A. Aquino-Cortez ${ }^{1}$, M. Valente $^{2}$, R. Toniolli ${ }^{1 *}$ \\ ${ }^{1}$ Universidade Estadual do Ceará \\ Av. Paranjana, 1700 \\ 60740-000 - Fortaleza, CE \\ ${ }^{2}$ Médica veterinária autônoma
}

\begin{abstract}
RESUMO
Em Barbalha, Ceará, foram realizados levantamentos de casos de cisticercose suína, teníase e neurocisticercose humana causadas por Taenia solium, e realizou-se uma pesquisa quanto aos sistemas de criação de suínos na região. De 85 suínos abatidos em abatedouro local 4,7\% apresentavam cisticercose, a maioria dos cisticercos localizava-se na língua e coração. Entre 2001 e 2004, das 302 criações de suínos denunciadas à vigilância sanitária, 96,6\% eram chiqueiros. A teníase, entre 1998 e 2003, correspondeu a $1,1 \%$ das verminoses diagnosticadas pela Secretária de Saúde Municipal. Entre 2001 e 2003, os casos de neurocisticercose humana corresponderam a 5\% das tomografias de crânio requisitadas nos hospitais da região.
\end{abstract}

Palavras-chave: Suíno, cisticercose, teníase, neurocisticercose humana, epidemiologia

\begin{abstract}
In Barbalha, municipal district of Ceará state, an assessment of cases of porcine cysticercosis, taeniasis and human neurocysticercosis caused by the Taenia solium was performed in order to evaluate the situation of these diseases. It was also carried out a research about the types of swine farms registered in the area. Considering the 85 swine slaughtered at the slaughterhouse of Barbalha, 4.7\% presented cysticercosis. Most cysticerci were located in the tongue and in the heart. Between 2001 and 2004, from 302 swine farms denounced to the Sanitary Surveillance, 9.6\% were pigsties. Taeniasis, between 1998 and 2003, corresponded to 1.1\% of the helminthes diagnosed by the Municipal Health Office. From 2001 to 2003, the cases of human neurocysticercosis corresponded to 5\% of the skull tomographies requested in the hospitals of the area.
\end{abstract}

Keywords: swine, cisticercosis, taeniasis, human neurocysticercosis, epidemiology

\section{INTRODUÇ̃̃O}

A cisticercose suína, a teníase e a neurocisticercose humana (NCC), causadas por Taenia solium, são problemas graves de saúde pública. Consideradas endêmicas em muitos países, nos quais a persistência dessas zoonoses está relacionada a fatores culturais e sócioeconômicos (Sarti et al., 2002), tais como, condições higiênico-sanitárias deficientes, sistemas precários de criação de suínos e não inspeção da carne, além da ausência de medidas de controle dessas doenças (Phiri et al., 2002).

A importância do complexo teníase/cisticercose para a saúde pública deve-se ao fato de o homem ser o hospedeiro definitivo de Taenia solium (Soulsby, 1997) ou o hospedeiro intermediário, ao abrigar a fase larval, Cysticercus cellulosae, principalmente no sistema nervoso central, desenvolvendo a neurocisticercose (Rey, 2002).

Recebido em 17 de dezembro de 2004

Aceito em 2 de fevereiro de 2007

*Autor para correspondência (corresponding author)

E-mail: toniolli@roadnet.com.br 
Dados de teníase e neurocisticercose humana podem ser obtidos, respectivamente, por meio de exames coprológicos e tomografia computadorizada (Pfuetzenreiter, 1997).

A determinação da prevalência da infecção em suínos por T. Solium é um importante requisito na maioria dos programas de controle da cisticercose (Sakai et al., 1998), e o método mais comum para o diagnóstico na espécie suína é a inspeção post-mortem das carcaças (Biondi et al., 1996). O diagnóstico da cisticercose em abatedouros, somado à informação da origem do animal, possibilita a definição de áreas de ocorrência da doença, bem como a sua qualificação para que possam ser executadas medidas de controle (Ungar e Germano, 1992).

Em Barbalha, sul do Ceará, são comuns a criação, a comercialização e o consumo de carne suína. No entanto, desconhece-se o tipo de criação da maioria dos animais destinados ao abatedouro municipal, assim como a prevalência da cisticercose suína, da teníase e da neurocisticercose humana na região, pois nunca se fez um estudo dessa situação. Este trabalho teve o objetivo de realizar um levantamento sobre os casos de cisticercose suína, teníase e neurocisticercose no município de Barbalha.

\section{MATERIAL E MÉTODOS}

O estudo foi realizado mediante inspeção de carcaças suínas quanto à presença de cisticercos, investigação dos principais tipos de criações de suínos existentes no município e levantamento de casos de teníase e neurocisticercose humanas.

Foram examinados 85 suínos abatidos no abatedouro municipal, no período de 6 de março a 7 de abril de 2004, todos provenientes de criações classificadas como chiqueiros. Após o abate, a carcaça era inspecionada minuciosamente observando-se a presença de cisticercos na língua, masseter, pernil, paleta, músculos intercostais e coração, locais considerados de predileção do cisticerco de $T$. solium na musculatura suína (Boa et al., 2002). Em uma planilha, eram identificadas e registradas todas as amostras com as respectivas alterações observadas nos órgãos e músculos. $\mathrm{Na}$ carcaça com cisticercose realizava-se a contagem dos cisticercos vivos e calcificados em cada músculo avaliado. O cisticerco foi considerado vivo ou viável quando se apresentava na fase vesicular, e calcificado, quando consistia em um nódulo sólido, mineralizado, de coloração esbranquiçada (Vianna et al., 2004).

O levantamento do número de pocilgas (criações com saneamento básico) e chiqueiros (sem esse benefício) existentes em Barbalha foi realizado a partir de dados da Vigilância Sanitária Municipal e contempla o período de 2001 a 2004. Os dados foram obtidos por meio de denúncias da população à Vigilância Sanitária sobre a existência de criações de suínos na zona urbana do município.

Foi realizado levantamento do número de casos de teníase em humanos, baseado nos exames coprológicos registrados e arquivados pela Secretária de Saúde do município entre 1998 e 2003, de acordo com Rey (2002). Dados relativos aos casos de neurocisticercose humana foram obtidos nos hospitais São Vicente de Paula e Santo Antônio, por meio de investigação dos laudos médicos de tomografias do crânio realizadas entre os anos de 2001 a 2003. Foi verificada a porcentagem de casos de neurocisticercose estimada na região de acordo com o sexo e a idade dos pacientes acometidos no período considerado.

Realizaram-se análises descritivas dos casos de cisticercose suína, dos tipos de criação de suínos e dos casos NCC de teníase. Para estudar a distribuição dos casos segundo o sexo e a idade utilizou-se o teste do qui-quadrado $(\mathrm{P}<0,05)$.

\section{RESULTADOS E DISCUSSÃO}

A inspeção das carcaças de 85 suínos revelou que quatro animais $(4,7 \%)$ apresentaram cisticercos. No mês anterior, a inspeção local havia detectado dois em 72 animais $(2,7 \%)$ com cisticercos (SIM, 2003).

A incidência de suínos infectados pode ser considerada elevada, visto a quantidade de animais criados na região e o curto período, um mês, de realização do levantamento. Esse percentual de animais parasitados constitui apenas uma estimativa do número real de suínos cisticercóticos abatidos na cidade, pois embora haja um abatedouro municipal, ainda existem 72 
matadouros clandestinos, que funcionam sob condições inadequadas de abate. Ressalta-se, ainda, que cerca de $50 \%$ dos marchantes, ao detectar o cisticerco na língua dos animais destinados ao abate, direcionavam esses para os mercados dos municípios vizinhos, onde não há inspeção veterinária de produtos de origem suína.

A distribuição dos cisticercos vivos e calcificados em cada músculo encontra-se na Tab. 1. Das quatro carcaças parasitadas, três (75\%) apresentaram cisticercos em todos os músculos inspecionados e uma, somente no coração. Nelas observaram-se 58 cisticercos. A língua, o coração e o pernil foram os locais com maior quantidade desse parasito (64\% do total). Embora a proporção de cisticercos tenha variado muito entre os órgãos e músculos, o maior percentual de cistos localizou-se nos órgãos rotineiramente examinados nas linhas de inspeção, como observado por Boa et al., 2002.

$\mathrm{Na}$ paleta e no diafragma a ocorrência foi de $6,9 \%$ e $8,6 \%$, respectivamente, diferente do mencionado por Soares et al. (2006), que encontraram $24,3 \%$ e $3,7 \%$, na mesma ordem de citação. Constata-se assim a importância da inspeção total e minuciosa, tanto nos órgãos recomendados como nos possivelmente infectados por T. solium.

O número de cisticercos calcificados foi maior (35) que o de viáveis (23), nos músculos avaliados $(\mathrm{P}<0,05)$, com exceção do coração (Tab. 1).

Tabela 1. Distribuição de Cysticercus cellulosae calcificados (CC) e viáveis (CV), segundo o músculo parasitado, em suínos inspecionados no Abatedouro de Barbalha, CE

\begin{tabular}{l|c|c|c|c} 
Músculo & CC & VC & Total & $\%$ \\
\hline Língua & 9 & 7 & 16 & 27,6 \\
Masseter & 4 & 2 & 6 & 10,3 \\
Paleta & 4 & 0 & 4 & 6,9 \\
Intercostais & 6 & 0 & 6 & 10,3 \\
Diafragma & 3 & 2 & 5 & 8,6 \\
Coração & 3 & 11 & 14 & 24,1 \\
Pernil & 6 & 1 & 7 & 12,1 \\
Total & 35 & 23 & 58 & \\
\hline
\end{tabular}

No município, dentro da área urbana, foram identificadas 302 criações suinícolas, sendo 10 pocilgas $(3,3 \%)$ e $292(96,7 \%)$ chiqueiros, de acordo com as denúncias verificadas junto à Vigilância Sanitária da cidade. Os números de pocilgas notificadas em 2001, 2002, 2003 e 2004 foram, respectivamente, $5,3,2$ e 0 ; os de chiqueiros, nos mesmos anos, foram 70, 80, 112 e 30, na mesma ordem de citação dos anos. Estes números são apenas estimativos, visto que existem criações que não são denunciadas.

Este resultado constitui-se num dado importante, já que a alta prevalência de cisticercose suína está diretamente correlacionada com o grande número de chiqueiros existentes em determinada região (Ngowi et al., 2004) e com a prevalência de teníase (Fane e Ooi, 1998).

A presença de humanos com teníase provocada pela ingestão de carne suína contaminada com a larva de T. solium constitui-se em alto risco para a cisticercose suína em populações rurais e centros urbanos (Ungar e Germano, 1992), sendo importante a investigação do número de casos dessa zoonose, em determinada região, como um indicativo da doença em suínos.

Segundo dados da Secretaria de Saúde do Município, de 1998 a 2003, essa parasitose correspondeu a $1,1 \%$ das verminoses diagnosticadas. Esse percentual está de acordo com a prevalência estimada da doença no Brasil, que pode variar de 0,3\% (Capuano et al., 2002) a 3\% (Epidemiology..., 1994). Segundo a Organização Panamericana de Saúde, a prevalência acima de $1 \%$ deve ser considerada alta (Yanez, 2001). A correlação entre o alto percentual de chiqueiros em Barbalha e os dados de prevalência de teníase humana deveria ser alta. No entanto, por ser uma doença de tratamento ambulatorial, não há registros de casos nos hospitais, o que leva a uma subnotificação da helmintose. Assim, o percentual de casos de teníase registrados pode ser considerado baixo, visto que constitui apenas uma subestimativa da doença.

Das 1792 tomografias de crânio realizadas entre os anos de 2001 a 2003, 90 (5\%) eram compatíveis com o diagnóstico de NCC. Foram registrados 16 casos em 2001, 44 casos em 2002 e 30 casos em 2003, e não houve diferença significativa no número de casos de $\mathrm{NCC}$ segundo o ano investigado $(\mathrm{P}>0,05)$. Com base nos laudos dos pacientes afetados, observou-se que a incidência da doença, no ano de 2002, foi 
mais alta em pacientes do sexo masculino, $61,4 \%$ versus $38,6 \%$. Essa diferença não foi detectada os anos de 2001 e 2003. Puetzenreiter e Ávila (1999) identificaram predominância em pacientes do sexo feminino. Essa relação entre sexos parece controversa: alguns estudos não evidenciaram diferenças entre os sexos (Chagas et al., 2003) e outros autores verificaram maior prevalência em pacientes do sexo masculino (Ito et al., 1998; Agapejev, 2003). Ao verificar a maior incidência em mulheres parasitadas, Fleury et al. (2004) sugeriram que fatores imuno- endocrinológicos poderiama influenciar na susceptibilidade e na patogênese da doença.

Apesar de estudos salientarem a importância da idade na prevalência da NCC (Cão et al., 1997; Lino et al., 1999), não houve diferença significativa entre as idades dos pacientes parasitados no ano de 2001 (Fig. 1). No ano de 2002, o percentual de pacientes com NCC nas idades de 1 a 10 anos foi menor que o de pacientes entre 21 e 50 anos e 51 e 80 anos.

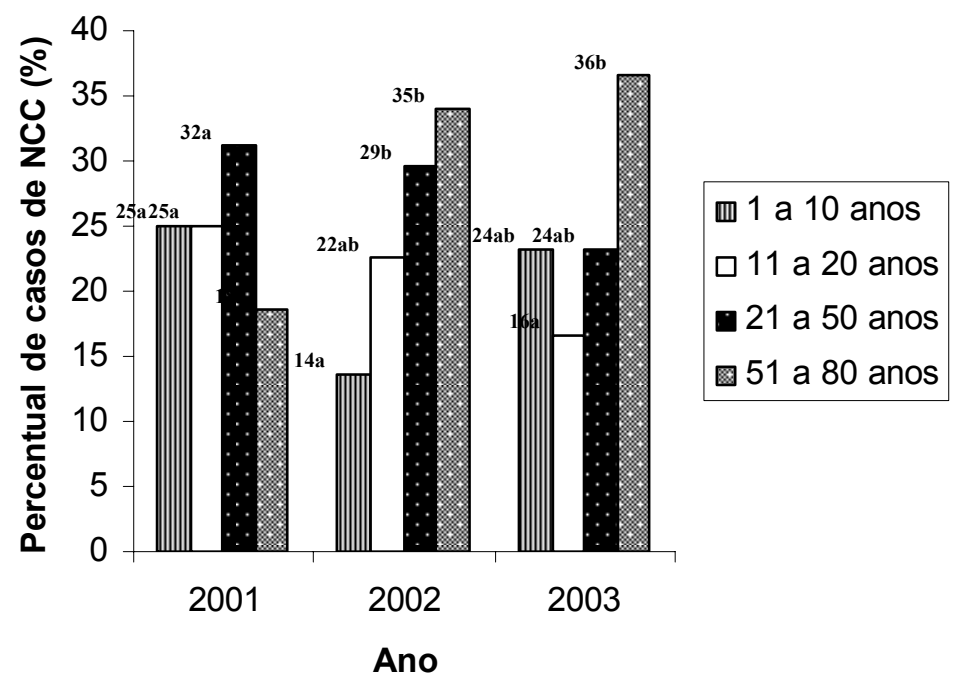

Figura 1. Percentual de casos de neurocisticercose, diagnosticados entre os anos de 2001 e 2003, de acordo com a faixa etária dos pacientes, no município de Barbalha, CE. Valores seguidos por letras distintas na coluna, dentro do ano, diferem entre si $(\mathrm{P}<0,05)$.

Em 2003, somente as faixas etárias de 11 a 20 e 51 a 80 anos diferiram entre si, isto é, o percentual de pacientes com idades entre 51 e 80 anos foi maior $(\mathrm{P}<0,05)$. A predominância de pacientes com NCC com idade acima de 50 anos também foi relatada por Gracia (1994) e Carrique-Mas et al. (2001). Os resultados obtidos reforçam a afirmação de que a NCC incide em qualquer faixa etária e em pacientes de ambos os sexos (Yamashita et al., 2003).

\section{CONCLUSÃO}

Conclui-se que o problema da cisticercose suína, e da teníase e neurocisticercose humanas é grave no município de Barbalha, CE, e assemelha-se aos resultados obtidos em outras regiões do país.

\section{REFERÊNCIAS BIBLIOGRÁFICAS}

AGAPEJEV, S. Neurocisticercose no Brasil. Arq. Neuropsiq., v.61, p.822-828, 2003.

BIONDI, O.f.; MUCCIOLO, R.G.; NUNES, C.M. et al. Immunodiagnosis of swine cysticercosis by indirect ELISA employing a heterologous antigen from Taenia crassiceps metacestode. Vet. Parasitol., v.64, p.261-266, 1996.

BOA, M.E.; KASSUKU, A.A.; WILLINGHAM, A.L. et al. Distribution and density of cysticerci of Taenia solium by muscle groups and organs in naturally infect local finished pigs in Tanzania. Vet. Parasitol., v.106, p.155-164, 2002.

CAO, W.; VAN DER PLOEG, C.P.B.; XU, J. et al. Risk factors for human morbidity: a populationbased case-control study. Epidemiol. Infec., v.119, p.231-235, 1997. 
CAPUANO, D.M.; OKINO, M.H.T.; BETYTINI, M.J.C.B. et al. Busca ativa de teníase e de outras endoparasitoses em manipuladores de alimentos no município de Ribeirão Preto, SP, Brasil. Rev. Inst. Adolfo Lutz, v.61, p.33-38, 2002.

CARRIQUE,-MAS, J.; IIHOSHI, N.; WIDDOWSON, M.A. et al. An epidemiological study of Taenia soluim cysticercosis in a rural population in the Bolivian Chaco. Acta Trop., v.80, p.229-235, 2001.

CHAGAS, M.G.L.; OLIVEIRA JUNIOR, A.D.; NETO, J.T. Manifestações clínicas da neurocisticercose na região do semi-árido do Nordeste brasileiro. Arq. Neuropsiq., v.61, p.398402, 2003.

FAN, P.C.; OOI, H.K. Taeniasis in Asia - an overview. Parasitol. Int., v.47, suppl., p.71-95, 2003.

FLEURY, A.; DESSEIN, A.; PREUX, P.M. et al. Symptomatic human neurocysticercosis: Age, sex and exposure factors relating with disease heterogeneity. J. Neurol., v.251, p.830-837, 2004.

GRACIA, A.K. Avaliação preliminar de casos de neurocisticercose de tomografia computadorizada em Curitiba, PR nos anos de 1992-1993. In: ENCONTRO CONE - SUL TENIASE/CISTICERCOSE - SEMINÁRIO LATINO AMERICANO SOBRE TENÍASE/CISTICERCOSE 1., 1994, Curitiba. Anais..., Curitiba, 1994.

ITO, Y.; MAK, J.; IDUKAWA, H. et al. Parasitol. Int., v.47, suppl., p.283-386, 1998.

LINO, J.; RUY, S.; REIS, M.A. et al. Occurrence of encephalic and cardiac cysticercosis (Cysticercus cellulosae) in necropsy. Rev. Saúde Publ., v.33, p.495-498, 1999.

NGOWI, H.A.; KASSUKU, A.A.; MAEDA, G.E.M. et al. Risk factors for the prevalence of porcine cysticercosis in Mbulu district, Tanzania. Vet. Parasitol., v.120, p.275-283, 2004.

EPIDEMIOLOGY y control de la
teniases/cisticercosis en America

PFUETZENREITER, M.R. Aspectos sócioculturais e econômicos de pacientes com diagnóstico preliminar de cisticercose cerebral em Lages, SC. Rev. Inst. Ciênc. Saúde. v.15, p.78-79, 1997.
PFUETZENREITER, M.R.; ÁVILA-PIRES, F.D. Fatores sócio-culturais e econômicos da cisticercose cerebral. Rev. Inst. Ciênc. Saúde, v.17, p.31-35, 1999.

PHIRI, I.K.; DORNY, P.; GABRIEL, S. et al. The prevalence of porcine cisticercose in Eastern and Southern provinces of Zambia. Vet. Parasitol., v.108, p.31-39, 2001.

REY, L. Bases da parasitologia médica. 2.ed. Rio de Janeiro: Guanabara Koogan, 2002. p.199-214.

SAKAI, H.; SONE, M.; CASTRO, D.M. et al. Seroprevalence of Taenia solium cysticercosis in a pigs a rural community of Honduras. Vet. Parasitol., v.78, p.233-238, 1998.

SARTI, G.E.; SCHANTZ, P.M.; AGUILLERA, J. et al. Epidemiologic observations on porcine cysticercosis in a rural commutiy of Michoacan state, Mexico. Vet. Parasitol., v.41, p.195-201, 2002.

SERVIÇO DE INSPEÇÃO MUNICIPAL DE BARBALHA, 2003.

SOARES, K.A.; SILVA, M.R.M.; POLETI, M.D. et al. Imunodiagnóstico da cisticercose em suíno experimentalmente infectado com ovos de Taenia solium, utilizando antígeno de escólex de Cysticercus cellulosae. Arq. Bras. Med. Vet. Zootec., v.58, p.15-20, 2006

SOULBY, E.J.L. Parasitologia y enfermidades parasitarias en los animales domésticos. 7.ed. México: Nueva Editorial Interamericana, 1997.

UNGAR, M.L.; GERMANO, P.M.L. Bovine cysticercosis prevalence in the state of São Paulo, Brazil. Rev. Saúde Publ., v.26, p.167-172, 1992.

VIANNA, L.G.; MACEDO, V.; COSTA, J.M. Cisticercose músculo-cutânea e visceral - doença rara? Rev. Inst. Med. Trop., v.33, 2004.

YAMASHITA, S.; MESQUITA, M.V.G.B.C.; MACHADO, G.C.M. et al. Cisticercose intramedular: relato de caso e revisão de literatura. Radiol. Bras., v.36, p.255-257, 2003.

YANEZ, G.C. Zoonoses de importância para a economia e saúde pública. In: REUNIÃO INTERAMERICANA, SOBRE SAÚDE E AGRICULTURA. 12., 2001, São Paulo. Anais... São Paulo: OPAS, 2001. 